\title{
Increased electric sail thrust through removal of trapped shielding electrons by orbit chaotisation due to spacecraft body
}

\author{
P. Janhunen \\ Finnish Meteorological Institute, Helsinki, Finland \\ Received: 20 November 2008 - Revised: 5 May 2009 - Accepted: 20 July 2009 - Published: 7 August 2009
}

\begin{abstract}
An electric solar wind sail is a recently introduced propellantless space propulsion method whose technical development has also started. The electric sail consists of a set of long, thin, centrifugally stretched and conducting tethers which are charged positively and kept in a high positive potential of order $20 \mathrm{kV}$ by an onboard electron gun. The positively charged tethers deflect solar wind protons, thus tapping momentum from the solar wind stream and producing thrust. The amount of obtained propulsive thrust depends on how many electrons are trapped by the potential structures of the tethers, because the trapped electrons tend to shield the charged tether and reduce its effect on the solar wind. Here we present physical arguments and test particle calculations indicating that in a realistic three-dimensional electric sail spacecraft there exist a natural mechanism which tends to remove the trapped electrons by chaotising their orbits and causing them to eventually collide with the conducting tethers. We present calculations which indicate that if these mechanisms were able to remove trapped electrons nearly completely, the electric sail performance could be about five times higher than previously estimated, about $500 \mathrm{nN} / \mathrm{m}$, corresponding to $1 \mathrm{~N}$ thrust for a baseline construction with $2000 \mathrm{~km}$ total tether length.
\end{abstract}

Keywords. Interplanetary physics (Instruments and techniques) - Magnetospheric physics (Solar wind interactions with unmagnetized bodies) General or miscellaneous (New fields (not classifiable under other headings))

\section{Introduction}

The electric sail (Janhunen, 2004; Janhunen and Sandroos, 2007 ) is a recently discovered, completely novel type of space propulsion system concept which uses the solar wind

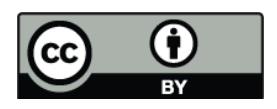

Correspondence to: P. Janhunen

(pekka.janhunen@fmi.fi) dynamic pressure for producing spacecraft propulsion. Technical development of the spinning electric sail (Janhunen, 2008a) commenced in 2006 , currently already being a long way towards actual realisation. The spinning electric sail uses the centrifugal force to deploy and stretch out a number of thin, long and conducting tethers from the spacecraft (Fig. 1). The tethers are then charged positively by an onboard electron gun so that their static electric field perturbs the trajectories of incident solar wind protons, resulting in a momentum transfer from the solar wind plasma stream to the tethers. A force law of the electric sail (Janhunen and Sandroos, 2007) was found from analytic considerations and one and two-dimensional particle-in-cell (PIC) simulations. It has been used to calculate successful and efficient mission trajectories in the solar system for realistic payloads and other spacecraft characteristics (Mengali et al., 2008a,b).

Since the earlier study (Janhunen and Sandroos, 2007) found that the number of trapped electrons is crucial in determining the width of the tether potentials and therefore the thrust per unit length, the subject of this paper is to analyse the source and loss mechanisms of trapped electrons in more detail, taking into account a realistic 3-D spacecraft geometry. As research methods we use theoretical considerations and test particle simulations, while self-consistent plasma simulations are deferred to future work.

The structure of the paper is as follows. After first briefly reviewing the original positive polarity electric sail concept, we analyse the origin of trapped electrons using a simple onedimensional box model, showing that the number of trapped electrons is independent of how rapidly the potential of the tether is turned on. Then we study trapped electron orbits, find their approximate constants of motion and show how the fact that the tethers are connected to a spacecraft "central hub" randomises the electron orbits so that the particles eventually collide with the wires and are lost. Finally we estimate the electric sail thrust based on the assumption of no trapped electrons and a local force balance on the "electropause" between electric pressure and solar wind dynamic

Published by Copernicus Publications on behalf of the European Geosciences Union. 


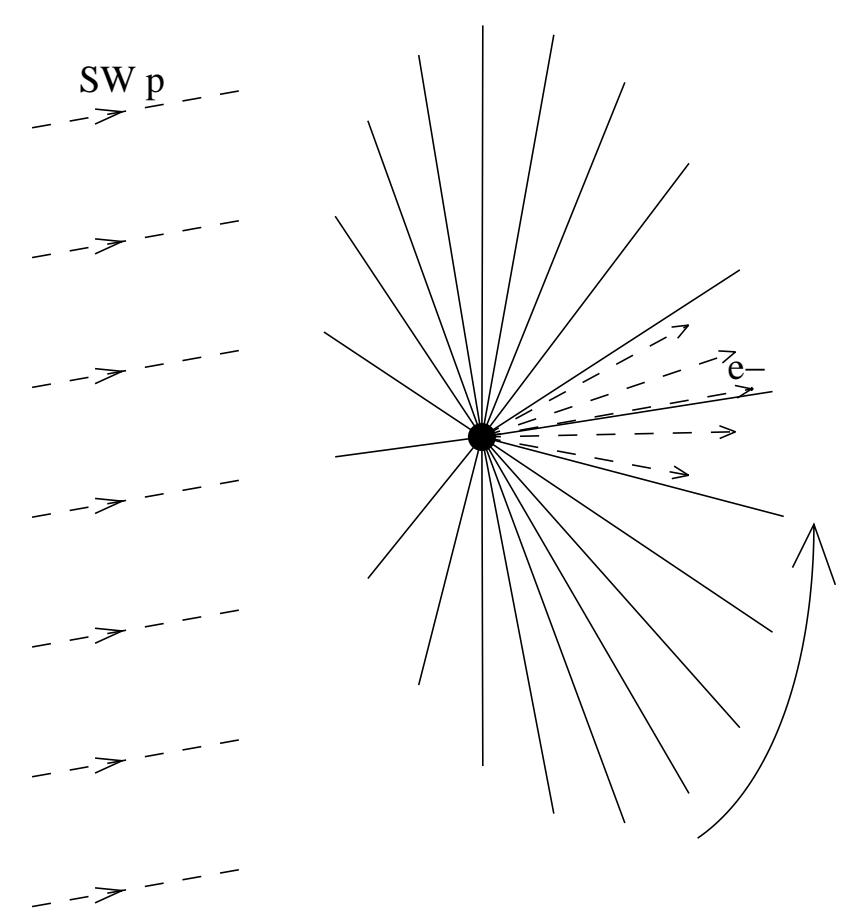

Fig. 1. Schematic description of the original spinning, positive polarity electric sail. Charged, centrifugally stretched tethers gather momentum from the solar wind. The charging is maintained by an electron gun mounted on the spacecraft (middle).

pressure. The thrust is found to be about five times larger than previously estimated by Janhunen and Sandroos (2007). The paper ends with a short discussion, summary and conclusions section.

\section{Electric sail basic design}

The electric sail (Fig. 1) consists of thin, long, conducting tethers which are kept positively charged by an onboard electron gun and stretched by their spinning (Janhunen, 2004; Janhunen and Sandroos, 2007; Janhunen, 2008b). The purpose of the electron gun is to pump out electrons from the system (spacecraft and tethers) so that a positive charge is left behind. Electrons are so lightweight that the momentum carried by the electron beam can be ignored, so that the electron gun can point in any direction in principle. Equally well there can also be more than one electron gun. The basic plasma physical problem is to estimate the thrust per unit tether length. The thrust will depend on the tether potential, tether radius (or rather, its effective electric radius introduced by Janhunen and Sandroos, 2007). Solar wind properties affecting the thrust are at least the density and speed, but according to Janhunen and Sandroos (2007) also the electron temperature.

The problem of evaluating the thrust appears to be essentially two-dimensional because the tethers are very long.

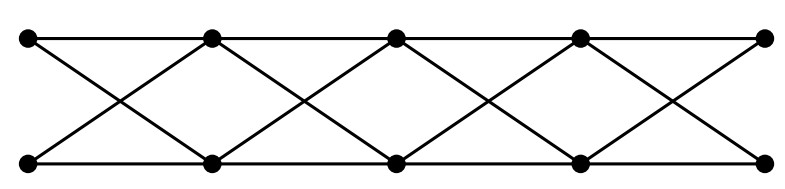

Fig. 2. A four-wire Hoytether. Wire bonding sites are shown by dots. The width of the structure is typically $2.5 \mathrm{~cm}$.

Only the component of the solar wind which is perpendicular to the tether plays a role, because the parallel equation of motion of the particles is trivial and not coupled to the other dynamical equations.

The problem of finding the potential pattern around the charged wire or tether was treated by Janhunen and Sandroos (2007) with a self-consistent time-domain particle-incell (PIC) plasma simulation. We now review their results in this section to set up notation and to get a starting point for our analysis.

In Janhunen and Sandroos (2007), it was found that a positively charged tether creates an electron sheath around itself where the potential of the cylindrical wire is approximately

$V(r)=V_{0} \frac{\ln \left[1+\left(r_{\mathrm{o}} / r\right)^{2}\right]}{\ln \left[1+\left(r_{\mathrm{o}} / r_{w}\right)^{2}\right]}$

where

$r_{\mathrm{o}}=2 \lambda_{D e}=2 \sqrt{\frac{\epsilon_{\mathrm{o}} T_{e}}{n_{\mathrm{o}} e^{2}}}$.

Here $\lambda_{D e}$ is electron Debye length, $T_{e}$ is the solar wind electron temperature (on average $T_{e}=12 \mathrm{eV}$ at $1 \mathrm{AU}$ ), $n_{\mathrm{o}}$ is the undisturbed solar wind electron density $\left(n_{\mathrm{o}}=7.3 \mathrm{~cm}^{-3}\right.$ on average at $1 \mathrm{AU}$ ) and $r_{w}$ is the (effective electric) radius of the tether, typically $r_{w} \approx 1 \mathrm{~mm}$. The effective electric radius (Janhunen and Sandroos, 2007) of the tether is larger than the physical radius of the wires $r_{w}^{\text {phys }} \approx 10 \mu \mathrm{m}$ of which the micrometeoroid-resistant multiline tether (Hoyt and Forward, 2001) is constructed (Fig. 2). The potential of the tether relative to the surrounding plasma is $V_{0}$. Equation (1) was found by Janhunen and Sandroos (2007) as a formula that fits well with the PIC simulation results. The time-domain PIC simulation produces a certain number of trapped electrons from the period when the potential is gradually turned on in the simulation. The shadowing effect of these trapped electrons is largely responsible for the form of Eqs. (1) and (2).

The solar wind ions experience the potential (1) and are deflected in their motion. In the frame of reference of the wire, the total energy of the ion is conserved, thus the particle has same speed after exiting the interaction region than it had originally. Because the direction of the velocity of the ion changes, however, the particle loses some of its momentum $\mathrm{x}$-component, where $x$ is the coordinate along the solar wind direction. This lost particle momentum is the reason 
for the thrust that the tether experiences. The momentum is transferred to the tether by an electric field due to piling up of ions on the sunward side of the tether (i.e., a positive charge cloud) and a corresponding ion void (negative charge cloud) on the antisunward side. Thrust is obtained because the electric field formed between the charge clouds (typically of the order $1 \mathrm{~V} / \mathrm{m}$ in magnitude) pushes the positively charged tether in the antisunward direction.

From Janhunen and Sandroos (2007), the force per unit length of the tether is given by

$$
\frac{d F}{d z}=\frac{K m_{p} n_{\mathrm{o}} v^{2} r_{\mathrm{o}}}{\sqrt{\exp \left[\frac{m_{p} v^{2}}{e V_{w}} \ln \left(r_{\mathrm{o}} / r_{w}\right)\right]-1}}
$$

where $v$ is the solar wind speed (typically $400 \mathrm{~km} / \mathrm{s}$ ). Here $K$ is a dimensionless calibration coefficient whose likely value is between 2 and 3 . A test particle calculation gives the value $K=3.09$ (Janhunen and Sandroos, 2007). Plasma particlein-cell (PIC) simulations give a result which is consistent with $K \approx 3$. However, Janhunen and Sandroos (2007) also showed that an analysis of the PIC results for different values for the electron temperature $T_{e}$ gives a result which is not quite consistent with the functional form of Eq. (3). One can explain away this inconsistency by postulating that due to numerical noise the effective value of the electron temperature in the PIC simulation electron sheath is higher than in the solar wind. If one assumes that this postulate is true, then the PIC simulation results are more consistent with $K \approx 2$ than with $K \approx 3$. Ultimately, only experiments made in space or in laboratory can give a certainty of the value of $K$. Again, we emphasise that also Eq. (3), in the same way as Eqs. (1) and (2), is inherited from the PIC simulation results of Janhunen and Sandroos (2007) which naturally include the trapped electron population from the turning-on phase of the potential.

We assumed above that the solar wind is unmagnetised. Neglecting the interplanetary magnetic field (IMF) is a good assumption because the ratio of the electron Larmor radius to the electron Debye length is

$\frac{r_{L e}}{\lambda_{D e}}=\sqrt{\frac{m_{e}^{2} v_{e}^{2} n_{\mathrm{o}}}{\epsilon_{\mathrm{o}} T_{e} B^{2}}}=\sqrt{\frac{m_{e}}{m_{i}}}\left(\frac{c}{v_{A}}\right)$.

At $1 \mathrm{AU}$, typically $v_{A} \approx 80 \mathrm{~km} / \mathrm{s}$ (corresponding to $B=$ $10 \mathrm{nT}$ and $n_{\mathrm{o}}=7.3 \mathrm{~cm}^{-3}$ ) while $\sqrt{m_{e} / m_{i}}=0.023$. Thus at $1 \mathrm{AU}, r_{L e} / \lambda_{D e} \approx 90 \gg 1$. Radially from the Sun, the quantity does not vary since it is proportional to the Alfvén speed $v_{A}$. The Alfvén speed does not vary because $\rho \sim 1 / r^{2}$ and $B \sim 1 / r$ in the equatorial plane. Thus the magnetic field can be neglected when considering electron motion in the sheath region. For ions, the Larmor radius is still larger by factor $\approx 70$ so they can be assumed to be unmagnetised as well.

The outward surface electric field on the tether wires is typically $100-200 \mathrm{MV} / \mathrm{m}$. This field is still not high enough that it could cause significant emission of ions or ion clusters from the surface. It is conceivable that some local microscopic protrusions may be torn off the surface by the electrostatic force. Such protrusions might exist on the metal surface originally or be caused by micrometeoroid cratering in space. If this happens, it should not cause any problems, since the expelled positively charged particles and fragments exit permanently to space. Only an electric field which is in the range $5-10 \mathrm{GV} / \mathrm{m}$ or even higher could be able to extract larger amounts of ions from a metal surface without the field being amplified by local protrusions.

As remarked above, the theory presented in Janhunen and Sandroos (2007) and which was reviewed in this section considers to tethers to infinitely long. While this is a good assumption in the sense that the tethers are much longer than the width of the potential structures, it ignores the fact that the tethers are at one end connected to the spacecraft which may modify the distribution function of trapped electrons. This was recognised also by Janhunen and Sandroos (2007) where it was speculated that by emitting low-frequency radio waves from the spacecraft, the trapped electron population could be heated so that the thrust which is proportional to square root of the electron temperature (Eq. 3) could then be increased. In this paper we concentrate on analysing the natural effect of the spacecraft on the trapped electron population. Our finding will be that trapped electrons will most likely be almost totally absent, which will also imply that Eqs. (1), (2) and (3) will no longer be valid, since they contain the shadowing effect of the trapped electrons which tends to underestimate the width of the potential structure and the electric sail force.

\section{Origin of trapped electrons}

When the potential of the tether is turned on so that the depth of the electron potential well increases with time, those electrons whose initial kinetic energy is very close to zero are trapped if the potential well deepens enough to prohibit their exit during the short time the particles spend inside the potential well. One might think that by turning the potential on slowly, the initial kinetic energy should be so extremely close to zero that almost no electrons would fulfil it and almost no electrons would become trapped. However, because the deepening time then also increases, it turns out that the number of electrons trapped is at least approximately independent of the time $t_{\mathrm{o}}$ that it takes to establish the potential.

We now show in a special case that the number of trapped electrons is independent of the deepening time $t_{0}$. Consider a toy model which is a one-dimensional linearly deepening box (slab) potential, defined by

$$
V(x, t)= \begin{cases}V_{0} \frac{t}{t_{\mathrm{o}}}, & 0 \leq x \leq b, \\ 0, & x<0 \text { or } x>b\end{cases}
$$


The traveltime of an electron through the box is $\tau=b / v_{\text {box }}$ where the speed inside the box $v_{\text {box }}$ is determined by

$\frac{1}{2} m_{e} v_{\mathrm{box}}^{2}=e V_{0} \frac{t}{t_{\mathrm{o}}}$,

assuming that, excluding a brief initial moment, the box depth is much larger than the particle's initial kinetic energy $(1 / 2) m_{e} v^{2}$. The particle becomes trapped by the deepening potential if the potential well deepens by more than the initial kinetic energy during the time $\tau$ the particle spends there, i.e., the condition for trapping is

$\frac{1}{2} m_{e} v^{2} \leq \frac{1}{2} m_{e} v_{\max }^{2} \equiv e V_{0} \frac{\tau}{t_{\mathrm{o}}}$.

Solving Eq. (7) for $v_{\max }$ and substituting $v_{\text {box }}$ as solved from Eq. (6) one obtains

$v_{\max }=\left(\frac{2 e V_{0}}{m_{e} t_{0} t}\right)^{1 / 4} \sqrt{b}$

The flux of electrons entering the box from one side is

$\Phi=\int_{0}^{v_{\max }} d v v f(v) \approx \frac{1}{2} v_{\max }^{2} f(0)$

where $f(v)$ is the distribution function and the approximation is good if the potential deepens slowly so that the window in velocity space which is sensitive for trapping is narrow enough that $f(v)$ does not appreciably change within that window. Concerning dimensionalities which we denote by $[.$.$] , notice that in 1-D case [f(v)]=\mathrm{s} / \mathrm{m}^{2}$ and $[\Phi]=$ is $1 / \mathrm{s}$.

The number of electrons trapped during the deepening time $t_{\mathrm{o}}$ of the potential is (notice that both sides of the potential well can absorb particles, hence the factor of 2)

$$
\begin{aligned}
N & =2 \int_{0}^{t_{0}} d t \Phi \\
& =f(0) \int_{0}^{t_{0}} d t\left(\frac{2 e V_{0}}{m_{e} t_{0} t}\right)^{1 / 2} b \\
& =2 f(0) \sqrt{\frac{e V_{0}}{2 m_{e} t_{\mathrm{o}}}} b 2 \sqrt{t_{\mathrm{o}}} \\
& =2 f(0) b \sqrt{\frac{2 e V_{0}}{m_{e}}} .
\end{aligned}
$$

Thus, the number of trapped electrons $N$ is independent of the deepening time $t_{\mathrm{o}}$ which cancels out of the expression.

We compute explicitly the density of trapped electrons $n_{\text {trap }}=N / b$ for a non-drifting Maxwellian source distribution

$f(v)=n_{\mathrm{o}} \sqrt{\frac{m_{e}}{2 \pi T}} \exp \left(\frac{-m_{e} v^{2}}{2 T}\right)$.
After a small calculation we obtain

$n_{\text {trap }}=n_{\mathrm{o}} \frac{2}{\sqrt{\pi}} \sqrt{\frac{e V_{0}}{T}}$.

For the electric sail, the potential depth $V_{0}$ is typically some tens of kilovolts while the electron temperature is only about ten volts, the the 1-D model would predict the trapped density to be some $\sim 30$ times higher than the solar wind electron density $n_{\mathrm{o}}$. That the trapped density grows as the square root of the potential was derived earlier by Gurevich (1968).

When the 1-D and 2-D PIC simulations of Janhunen and Sandroos (2007) were run with different potential turning on times $t_{\mathrm{o}}$, the results were found not to depend on $t_{\mathrm{o}}$. Thus there is simulation evidence that the result that the number of trapped electrons is independent of $t_{\mathrm{o}}$ is valid for the physically relevant 2-D wire potential case, in the same way as we showed in this section it to hold analytically for the 1-D toy model.

\section{Constants of motion of trapped electrons}

We ended the previous section with the assertion that the speed at which the potential of the tether is turned on does not affect the number of trapped electrons resulting from the process. In other words, the birth of a certain number of trapped electrons which orbit the wire and which decrease the electric sail force by their shadowing effect appears to be a fundamental phenomenon which is not possible to get rid of. We shall now aim at investigating what happens to these trapped electrons orbiting the wire once a quasi-stationary situation has been reached. We start by finding the constants of motion of the electrons in a symmetric potential of the wire where shielding effect is also qualitatively taken into account.

Consider an electron which is trapped in an infinitely long, static and cylindrically symmetric potential $V(r)$ around a tether. We assume that the potential is a shielded version of the vacuum wire potential (Janhunen and Sandroos, 2007),

$V(r)=\frac{V_{0} \ln \left[1+\left(r_{\mathrm{o}} / r\right)^{2}\right]}{2 \ln \left(r_{\mathrm{o}} / r_{w}\right)}$

where $r_{\mathrm{o}}$ is the shielding distance and $r_{w}\left(r_{w} \ll r_{\mathrm{o}}\right)$ is the electric radius of the tether, which for a simple cylindrical wire coincides with its physical radius. We use cylindrical coordinates $(r, \varphi, z)$. Notice that Eq. (13) is used here in the qualitative sense only and that the shielding distance parameter $r_{\mathrm{O}}$ appearing in it can have any value and is not necessarily given by Eq. (2). For our purpose here, we could in principle use even the vacuum potential $V(r)=V_{0} \ln \left(r_{\mathrm{o}} / r\right) / \ln \left(r_{\mathrm{o}} / r_{w}\right)$ (Eq. 1 of Janhunen and Sandroos, 2007), but expression (13) is more convenient because it tends to zero for $r \rightarrow \infty$ while being close to the vacuum potential for $r \ll r_{0}$.

The speed of the particle along the tether $v_{z}$ stays constant in time, as does its total energy $W_{\text {tot }}=W_{k}^{\|}+W_{k}^{\perp}-e V(r)$ 

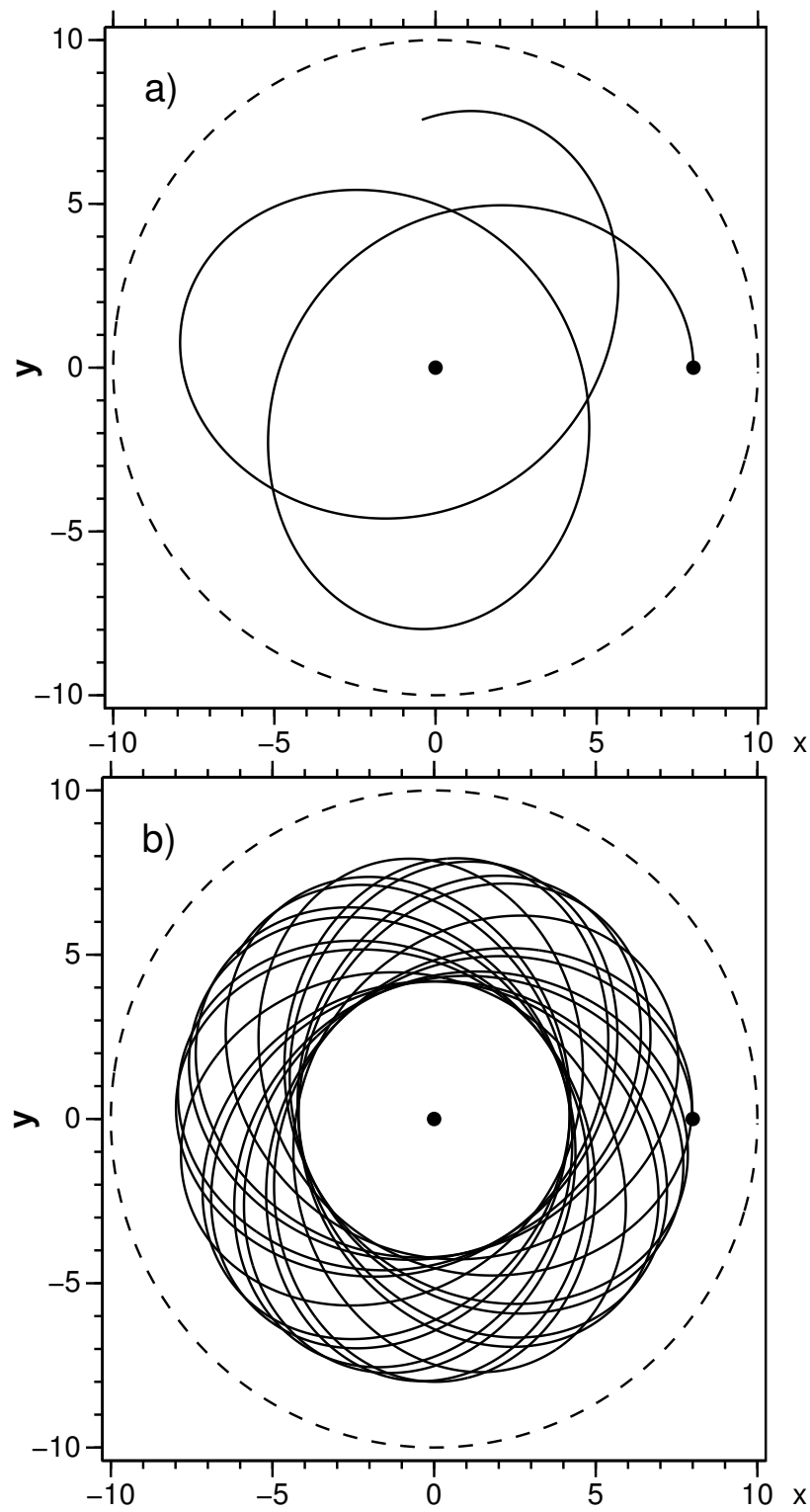

Fig. 3. Example electron orbit in potential (13) with $V_{0}=20 \mathrm{kV}$, $r_{\mathrm{o}}=19 \mathrm{~m}$ and $W_{\text {tot }}^{\perp}=-1.5 \mathrm{keV}$, after $5 \mu$ s integration (a) and after $40 \mu$ s integration (b).

where $W_{k}^{\|}=(1 / 2) m v_{z}^{2}$ and $W_{k}^{\perp}=(1 / 2) m\left(v_{r}^{2}+v_{\varphi}^{2}\right)$. Because $W_{\text {tot }}$ and $W_{k}^{\|}$are both conserved, the total perpendicular energy $W_{\text {tot }}^{\perp}=W_{k}^{\perp}-e V(r)$ is also a constant of motion. Finally, since the potential does not depend on $\varphi$, the angular momentum per unit mass of the particle $L_{z}=r v_{\varphi}$ is conserved as well.

In the perpendicular plane, the particle describes a quasielliptic orbit (Fig. 3) which is usually, however, far from being closed, i.e. the perihelion angle changes rapidly. We can agree, for example, that we start the integration from the particle's aphelion where momentarily $v_{r}=0$. Furthermore, we

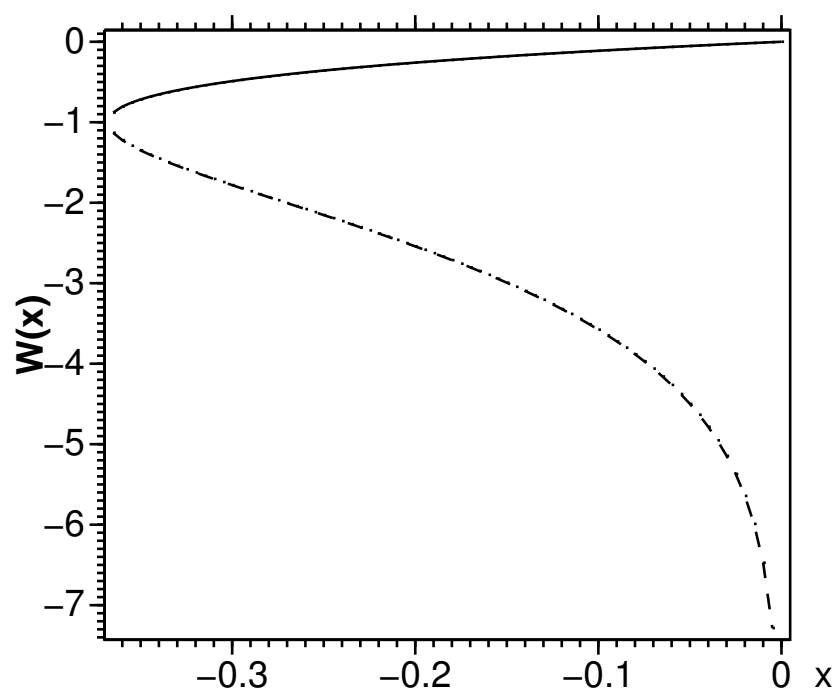

Fig. 4. Lambert's $W$-function $W(x)$ for negative argument. Both principal (solid) and non-principal (dashed) branches are shown.

can agree that the aphelion occurs at some fixed $\varphi$ e.g. $\varphi=0$, so that the particle's initial state $\left(r, \varphi=0, v_{r}=0, v_{\varphi}\right)$ contains two free parameters $r$ and $v_{\varphi}$. Fixing these parameters fixes the perpendicular energy $W_{\text {tot }}^{\perp}=(1 / 2) m v_{\varphi}^{2}-e V(r)$ and the angular momentum $L_{z}=r v_{\varphi}$.

For a given $L_{z}$ and $W_{\text {tot }}^{\perp}$, what is the radial range $\left[r_{\min }, r_{\max }\right]$ where the particle moves? This question admits an analytic solution in terms of Lambert's $W$-function, also called product $\log$ function (Fig. 4). For any $x$, the function $W(x)$ is defined as the solution of the equation $x=W(x) e^{W(x)}$. For $x \geq 0$ the solution is unique, but for $-1 / e \leq x<0$ the function has two branches called the principal and non-principal branch, while for $x<-1 / e$ the function is complex-valued. Omitting the derivation, the result for the particle's radius is

$r=\frac{r_{\mathrm{o}}}{\sqrt{-1-W\left(-a e^{-a-b}\right) / a}}$

where

$$
\begin{aligned}
a & =\frac{V_{L}}{V_{0}} \ln \left(r_{\mathrm{o}} / r_{w}\right) \\
b & =\frac{2 W_{\mathrm{tot}}^{\perp}}{e V_{0}} \ln \left(r_{\mathrm{o}} / r_{w}\right) \\
V_{L} & =\frac{m_{e}}{e}\left(\frac{L_{z}}{r_{\mathrm{o}}}\right)^{2}
\end{aligned}
$$

We call the dimensionless number $V_{L} / V_{0}$ the angular momentum parameter because it is proportional to $L_{z}$. Lambert's $W$-function is here evaluated for negative argument where it has two real-valued branches. The principal (less negative) branch corresponds to $r_{\max }$ and the non-principal (more negative) branch to $r_{\min } . W(x)$ is real-valued for 


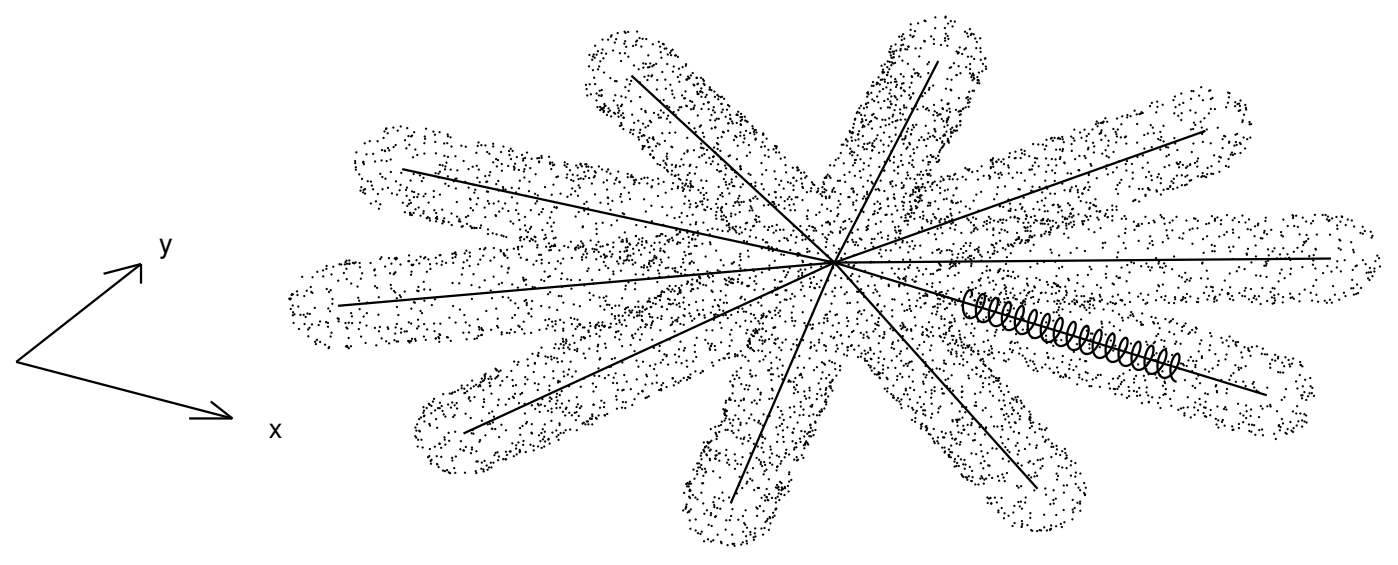

Fig. 5. Schematic description of the starfish-like electrostatic potential structure around positively charged electric sail tethers. An example electron which moves along and spirals around one of the tethers is also shown.

$x \geq-1 / e$ only. For a fixed $W_{\text {tot }}^{\perp}$, this restricts the possible values of $L_{z}$ so the one must have:

$\frac{V_{L}}{V_{0}} \leq \frac{V_{L}^{\max }}{V_{0}}=\frac{-W\left(-e^{b-1}\right)}{\ln \left(r_{\mathrm{o}} / r_{w}\right)}$.

At the maximal angular momentum parameter $V_{L}^{\max } / V_{0}$, the argument of the $W$-function is equal to $-1 / e$, its value is -1 and its two branches coincide. At this point the orbit is a circle in the perpendicular plane with radius

$r_{\text {circ }}=\frac{r_{\mathrm{o}}}{\sqrt{-\frac{1}{W\left(-e^{b-1}\right)}-1}}$

where the principal branch of $W$ must now be chosen because the non-principal one whose values are less than -1 would yield an imaginary solution for $r_{\text {circ }}$.

Summarising this section, we showed that corresponding to the fact that the potential $V(r, \varphi, z, t)$ actually depends only on $r$, so that the angular momentum $L_{z}$, the parallel kinetic energy $W_{k}^{\|}$and the total energy $W_{\text {tot }}$ are constants of motion of the trapped electron. For given $V_{0}, W_{k}^{\|}$and $W_{\text {tot }}$ there exists a maximal value of the angular momentum, the corresponding trajectory of which describes a circle in the perpendicular plane. Smaller values of the angular momentum correspond to orbits with nonequal perigee and apogee distance $r_{\min }$ and $r_{\max }$. For general angular momentum, the quantities $r_{\min }$ and $r_{\max }$ can be expressed analytically in terms of Lambert's $W$-function.

\section{Chaotisation of trapped orbits in 3-D case}

As shown in the previous section, a trapped electron orbits its tether while moving along it at some parallel speed (Fig. 5). In addition to the conserved total energy, the perpendicular dynamics is characterised by two other constants of motion, the total perpendicular energy $W_{\text {tot }}^{\perp}$ which is negative because the electron is bound and the tether-aligned component $L_{z}$ of the angular momentum. Initially the parallel speed is of the order of the solar wind thermal speed which is $\sim 1500 \mathrm{~km} / \mathrm{s}$ so that it takes about $10 \mathrm{~ms}$ for an electron to traverse the total length of a typical $20 \mathrm{~km}$ long electric sail tether. At the tip of the tether the electron is reflected back towards the spacecraft.

Coulomb collisions can slowly alter the trapped electron population, but the timescales involved are weeks or months in case of the solar wind electric sail. Radiative losses can also remove energy from the trapped electrons, but also this process is extremely slow. However, it takes only $\sim 0.02 \mathrm{~s}$ for an electron at parallel velocity $1500 \mathrm{~km} / \mathrm{s}$ to travel back and forth a $20 \mathrm{~km}$ long tether. Thereafter the electron arrives at the vicinity of the spacecraft where the potential structure is three-dimensional and starfish-shaped. The electron does not stay in the vicinity of the spacecraft for long, but continues its path along one of the tethers, which may or may not be the same tether along which it arrived.

When interacting with the spacecraft, the electron's angular momentum does not stay constant. The total energy is still conserved, but a repartitioning of the parallel and perpendicular energy occurs. To see how this happens in detail, we use a test particle simulation.

To make the the test particle calculation more efficient, we make the following simplifications: (1) instead of several tethers, we use two perpendicularly crossed tethers, (2) one of the tethers ( $y$-directed) is ten times shorter than the $x$ directed one, (3) the longer ( $x$-directed) tether is $1 \mathrm{~km}$ long rather than $20 \mathrm{~km}$ long. For each pass of the particle along the $x$-directed tether, the particle's constants of motion (angular momentum, parallel speed, minimum and maximum distance from the tether, total perpendicular energy) are measured by averaging when it is located at $500-900 \mathrm{~m}$ distance from the spacecraft. Each tether in the calculation is surrounded by a shielded potential structure of the form (1) 


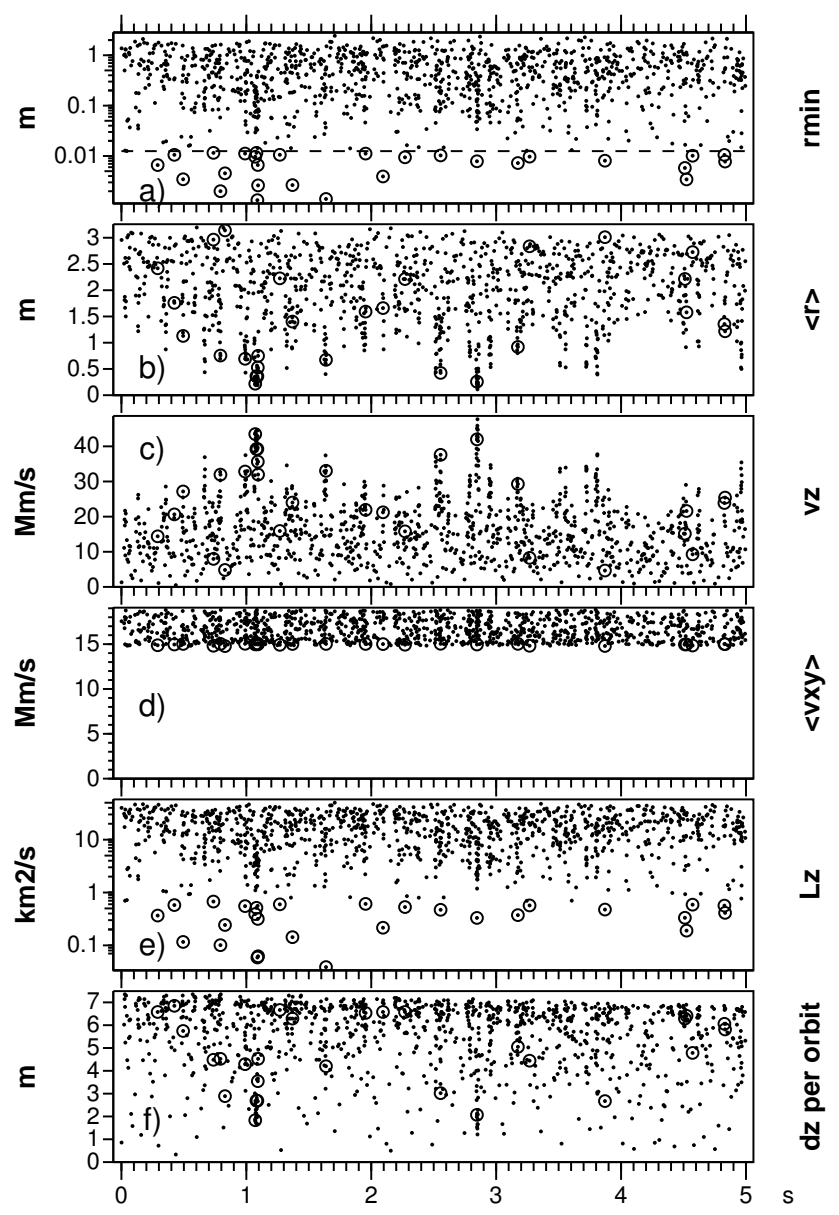

Fig. 6. Tether passes of a test particle calculation: (a) minimum distance from tether, (b) average distance from tether, (c) parallel velocity, (d) orbit-averaged perpendicular velocity, (e) angular momentum component along tether, (f) how much particle advances itself along tether while circling around it for one orbit. Points with minimum distance less than $1.25 \mathrm{~cm}$ are circled. The $1.25 \mathrm{~cm}$ limit is indicated by in dashed line in panel (a). The time axis has been scaled to correspond to $20 \mathrm{~km}$ long tethers.

where the shielding parameter $r_{\mathrm{o}} \approx 20 \mathrm{~m}$, so at $500 \mathrm{~m}$ distance from the tether crossing the particle is effectively free from influence of the spacecraft.

During the test particle calculation, we detect the particle's passage along the $x$-directed (longer) tether and record its constants of motion during each such passage. Figure 6 shows the results of one such calculation. The panels show the minimum radius $r_{\text {min }}$, mean radius $\langle r\rangle$, parallel speed $v_{z}$, mean perpendicular speed $\left\langle v_{x y}\right\rangle$, angular momentum $L_{z}$ and how much the particle progresses along the tether between successive distance minima. The time axis has been edited so that it corresponds to a $20 \mathrm{~km}$ long tether. That is, each point in Fig. 6 corresponds to one passage of the electron along a tether and the time intervals between points correspond to correct traveltimes of the electron along a full-length $(20 \mathrm{~km}$ long) tether.
We see from Fig. 6 that the angular momentum $L_{z}$ and the parallel velocity $v_{z}$ are well randomised by each encounter with the spacecraft's 3-D potential structure. Notice also that the typical parallel speed becomes much higher than the original $1.5 \mathrm{Mm} / \mathrm{s}$. This happens because the spacecraft-induced randomisation causes a redistribution of parallel and perpendicular energy so that speeds parallel and perpendicular to the tether are usually of the same order of magnitude. This means that after the first randomisation the electron usually completes its trip along the tethers even faster than the $0.02 \mathrm{~s}$ that was estimated above.

The baseline electric sail tether construction is shown in Fig. 2. The multiline tether whose typical width is $2.5 \mathrm{~cm}$ is so constructed for enhanced micrometeoroid survivability of the tether (Hoyt and Forward, 2001). The tether wires are about $20 \mu$ thick so that the wires cover about $1 / 1000$ of the tether area. This means that if an electron comes closer to than about $1 \mathrm{~cm}$ of the tether axis when it orbits the tether, at each orbit there is a $\sim 1 / 1000$ probability that it hits a tether wire and gets removed. From the bottom panel of Fig. 6 we can infer that since the progress distance per orbit is less than $10 \mathrm{~m}$, the particle completes several thousand orbits when traversing back and forth a $20 \mathrm{~km}$ tether. Thus once inserted by the spacecraft into an orbit which has a low enough $L_{z}$ so that $r_{\min }<1.25 \mathrm{~cm}$, the electron will probably collide with the tether before its next visit to the spacecraft.

Data points corresponding to electron passages with $r_{\min }<1.25 \mathrm{~cm}$ are circled in Fig. 6. In this calculation which corresponds to $-3 \mathrm{keV}$ total energy in the field of a $V_{0}=20 \mathrm{kV}$ tether with electric radius $r_{w}=1 \mathrm{~mm}$, the number of those passages is 28 which would give an average $0.18 \mathrm{~s}$ lifetime for the electron. Close to the tether, our numerical model in principle breaks down since the electric field of the Hoytether (Fig. 2) is no longer radially symmetric at close distance. The deviation of the field from radial symmetry might cause additional small changes of the particle's $L_{z}$, so that the true lifetime of the electron might even be somewhat less than our numerical estimate.

With what probability does the spacecraft interaction generate states with different angular momenta $L_{z}$ and minimum radii $r_{\min }$ ? Figure 7 shows that $L_{z}$ and $r_{\min }$ are nearly linearly correlated so one can consider either one, while Fig. 8 displays the distribution of $L_{z}$ values in the 5-s long calculation containing 1170 tether traversals. We see that the $L_{z}$ probability distribution is nearly linearly falling from zero to the maximum allowed $L_{z}$. The maximum angular momentum (Eq. 18) depends on the particle's total energy and it corresponds to circular orbit whose radius is given by Eq. (19). Finally, Fig. 9 shows a scatter plot of the particle's parallel kinetic energy $W_{k}^{\|}$and $L_{z}$. The maximal angular momentum is reached for a particle which orbits the tether circularly and whose parallel speed is small so that most of its kinetic energy is of the perpendicular type. 


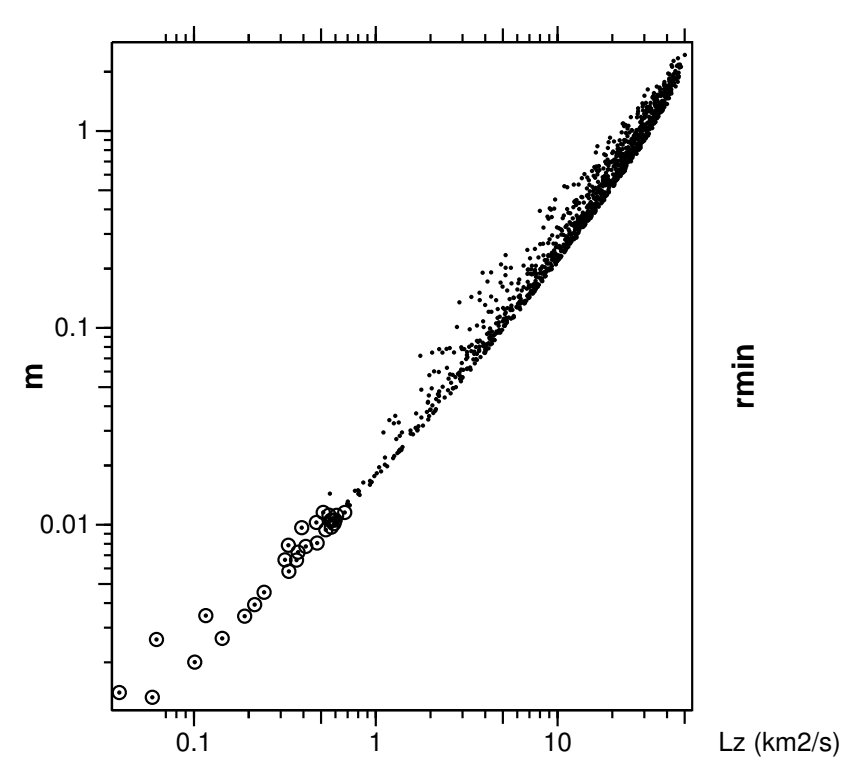

Fig. 7. Scatter plot of particle's angular momentum $L_{z}$ and minimum radial distance $r_{\min }$ in the test particle simulation.

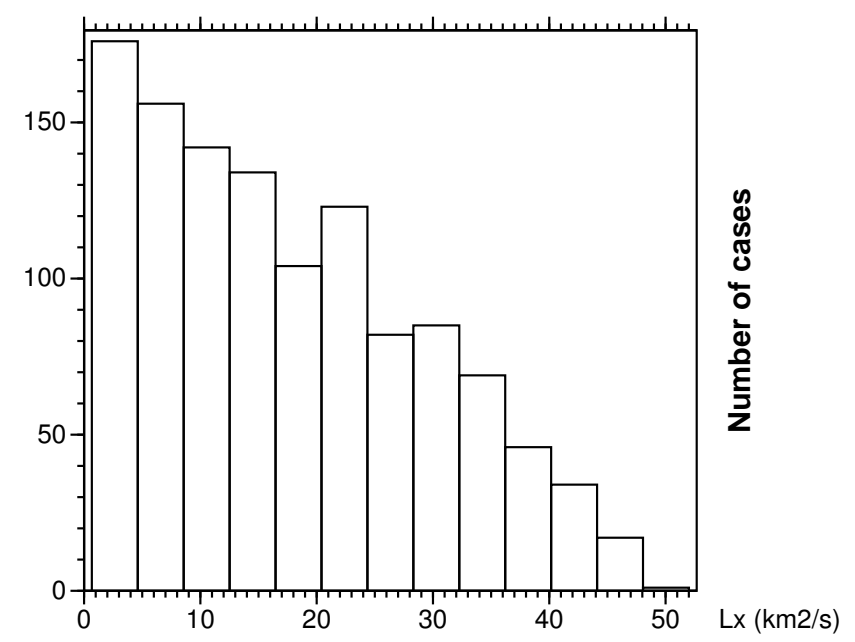

Fig. 8. Distribution of electron's angular momentum $L_{z}$ in the test particle simulation.

The mean parallel speed of the particle is $17 \mathrm{Mm} / \mathrm{s}$, corresponding to $820 \mathrm{eV}$ energy which is about the same as the kinetic energy of a particle moving in the maximal circular orbit, Eq. (19). The mean perpendicular speed is about the same as the mean parallel speed.

\section{Trapped electron lifetime}

We are now ready to go back to analytic formulas and to develop an approximate answer to the question of the lifetime of a trapped electron against removal by tether collisions.

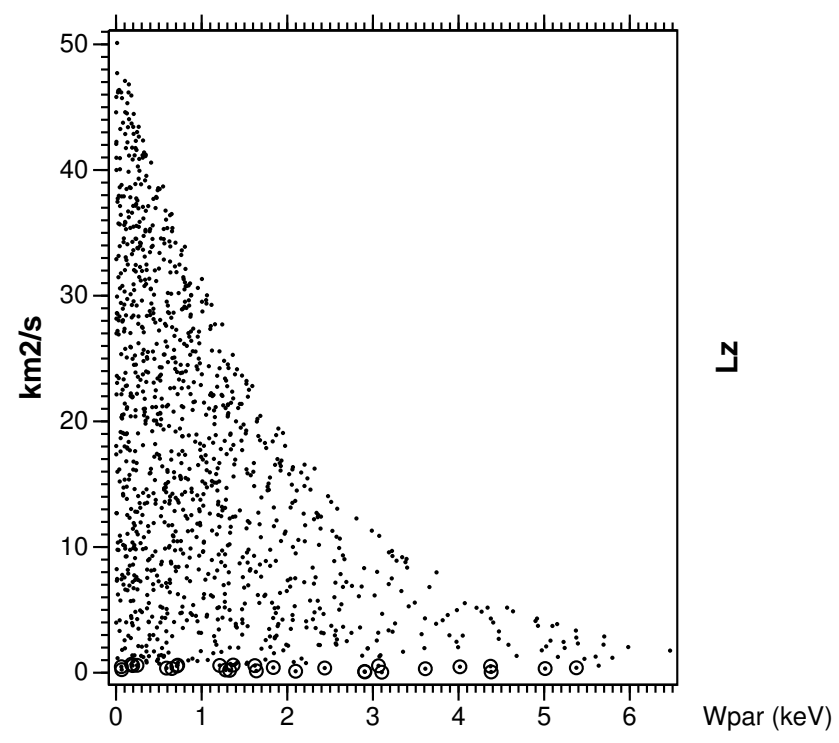

Fig. 9. Scatter plot of particle's parallel kinetic energy $W_{k}^{\|}$and angular momentum $L_{z}$ in the test particle simulation.

Consider a trapped electron with total energy $W_{\text {tot }}$. We shall first compute the probability $P_{1}$ that the particle emerges from the spacecraft interaction with an orbit which is on collision course with the tether wires, i.e. that its $r_{\text {min }}$ is smaller than half of the tether width $w_{t}$. Based on Figs. 8 and 7, we model the $r_{\min }$ distribution as a triangular (linearly decreasing) one. The largest allowed value of $r_{\min }$ is given by $r_{\text {circ }}$ (Eq. 19) with $W_{k}^{\|}=0$. The probability density $d P_{1} / d r_{\min }$ at $r_{\min } \approx 0$ is then equal to $2 / r_{\text {circ }}$ because of the triangular distribution assumption. The probability $P_{1}$ is then obtained as

$P_{1}=\frac{d P_{1}}{d r_{\min }} \frac{w_{t}}{2}=\frac{w_{t}}{r_{\text {circ }}}$.

We then estimate the probability $P_{2}$ that the electron actually hits a tether wire. In the numerical calculation of the previous section this probability was essentially unity, but if the particle's total energy is less negative, its orbiting time around the tether is longer and a collision may not take place. The test particle calculations above showed that the average parallel and perpendicular velocities are equal and both are close to the velocity of an electron orbiting at the maximal circular orbit. Thus the velocities are equal to

$\left\langle v_{\|}\right\rangle=\left\langle v_{\perp}\right\rangle=\sqrt{\frac{2\left(e V\left(r_{\text {circ }}\right)+W_{\text {tot }}^{\perp}\right)}{m_{e}}}$.

If and when trapped particles are removed, first near the tether and then progressively to higher radial distances also, the tether electric field becomes less shielded so that $r_{\mathrm{o}}$ effectively increases. At any moment of time, a typical electron has $r_{\text {circ }} \sim r_{\mathrm{o}}$. When $r_{\mathrm{o}}=r_{\text {circ }}$, a small calculation based on Eq. (19) then shows that the total energy $W_{\text {tot }}^{\perp}$ is then $28 \%$ 


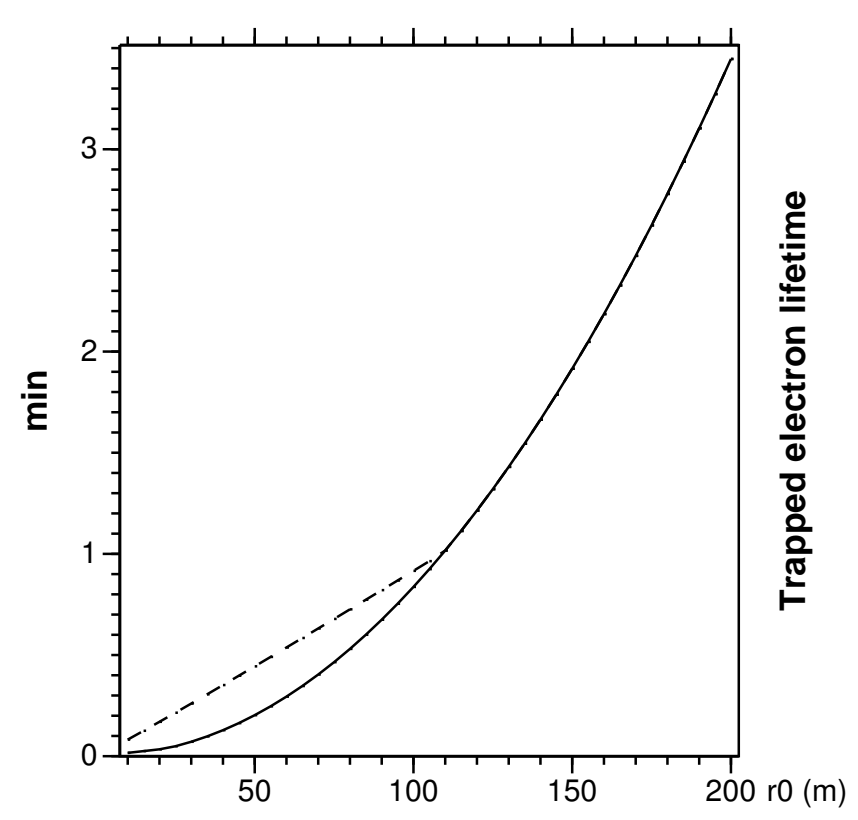

Fig. 10. Trapped electron lifetime (Eq. 23) as function of shielding distance $r_{\mathrm{O}}$ for $20 \mathrm{~km}$ (solid) and $100 \mathrm{~km}$ (dashed) tethers.

of the potential energy $-e V\left(r_{\text {circ }}\right)$. Therefore, in a rough calculation we may omit the term $W_{\text {tot }}^{\perp}$ in Eq. (21). For each orbit, since the parallel and perpendicular speeds are on average equal, the particle advances a distance $2 \pi r_{\text {circ }}$ so that the number of orbits per $2 L$ back-and-forth travel distance along the $L$-length tether is $L /\left(\pi r_{\text {circ }}\right)$. For each orbit, the probability of a collision is given by the areal fraction of wires in the tether area (Fig. 2). This single-orbit collision probability is equal to $2 r_{w}^{\text {phys }} f_{\mathrm{mul}} / w_{t}$ where $r_{w}^{\text {phys }}$ is the physical radius of the wire and $f_{\mathrm{mul}}$ is the effective tether multiplicity $\left(f_{\text {mul }}=4.3\right.$ for the tether shown in Fig. 2). All in all, the probability $P_{2}$ is then (recall that we assume $r_{\mathrm{o}}=r_{\text {circ }}$ )

$P_{2}=\min \left(1, \frac{L}{\pi r_{\mathrm{o}}} \frac{2 r_{w}^{\text {phys }} f_{\mathrm{mul}}}{w_{t}}\right)$.

The frequency $f$ at which the particle visits the spacecraft "hub" is equal to $2 L / v_{\|}$where $v_{\|}$is obtained from (21) with the $W_{\text {tot }}^{\perp}$ term omitted as motivated above. The electron lifetime is then $\tau=1 /\left(P_{1} P_{2} f\right)$ which is our final result. By substituting the variables, the lifetime can be finally written as

$\tau=\sqrt{\frac{8}{\ln 2} \ln \left(\frac{r_{\mathrm{o}}}{r_{w}}\right)} \frac{r_{\mathrm{o}} L}{w_{t} v_{e}^{\max }} \max \left(1, \frac{\pi}{2 f_{\mathrm{mul}}} \frac{r_{\mathrm{o}} w_{t}}{L r_{w}^{\text {phys }}}\right)$

where $v_{e}^{\max }$ is the speed of the electron when raised to potential $V_{0}, v_{e}^{\max }=\sqrt{2 e V_{0} / m_{e}}$.

Equation (23) expresses the lifetime of a typical electron when the potential structure shielding width is $r_{\mathrm{o}}$ in terms of technical parameters $L, r_{w}, r_{w}^{\text {phys }}, f_{\mathrm{mul}}, w_{t}$ and $V_{0}$ of the electric sail. The lifetime is plotted in Fig. 10 for 20 and $100 \mathrm{~km}$

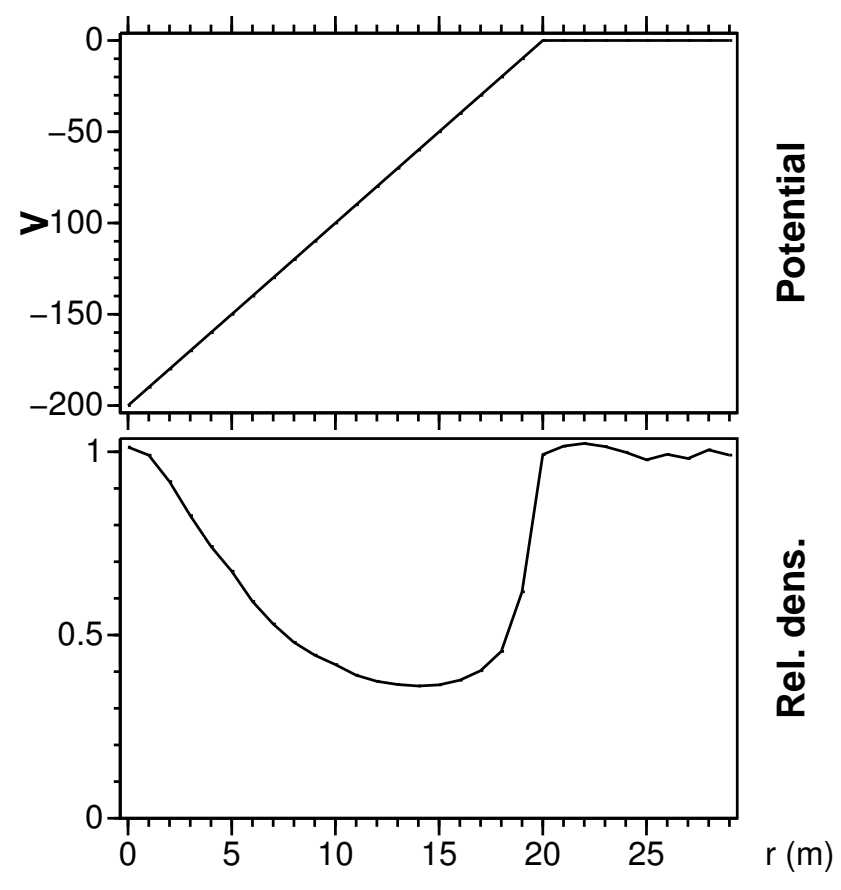

Fig. 11. Test particle calculation where 4000 electrons (temperature $12 \mathrm{eV}$ ) were launched into a 20-m wide and $200 \mathrm{~V}$ deep, linearwalled potential well (upper panel). Lower panels shows the resulting electron density.

long tethers. For high $r_{\mathrm{o}}$, the probability $P_{2}$ is smaller than unity, the lifetime proportional to $r_{\mathrm{o}}^{2}$ and independent of $L$. For small $r_{\mathrm{o}}, P_{2}$ becomes unity so that the lifetime depends linearly on $r_{\mathrm{o}}$. Thus increasing the tether length increases the electron lifetime up to certain value of $r_{0}$, but not dramatically and not for large $r_{\mathrm{o}}$. The most important practical implication of Eq. (23) and Fig. 10 is that the trapped electron lifetime is so short (order of few minutes) that there is ample time for their natural removal to take place in the context of electric sail operation. In the next section we shall estimate the electric sail thrust when trapped electrons are absent.

\section{Thrust estimation}

What is the shape and size of the potential structure around a charged tether if there are no trapped electrons at all? In this situation, external electrons which are moving through the stationary potential in hyperbolic (positive energy) orbits are the ones that must take care of screening the charge of the tether inside. In a two-dimensional potential structure case one can establish an analytic upper bound to the density of these electrons. At any point $(x, y)$ inside the potential structure, consider a point $P$ in 4-D phase space, $P=\left(x, y, v_{x}, v_{y}\right)$. A backward integration of the particle orbit from $P$ either ends up being far outside the potential structure or stays forever inside the structure forming a 
periodic or quasi-periodic orbit. If the point is far outside, then according to Liouville's theorem the value of the distribution function at $P, f(P)=f\left(x, y, v_{x}, v_{y}\right)$, is equal to the value of the distribution function $f_{\mathrm{o}}\left(\mathbf{v}^{\prime}\right)$ in the external, uniform plasma, where $\mathbf{v}^{\prime}$ is the original velocity of the particle produced by the backward trajectory integration. If backward integration stays forever inside the potential structure, then by assumption of no trapped electrons, the value of the distribution function $f(P)$ must be zero. We can cover both cases if we write

$f\left(x, y, v_{x}, v_{y}\right)=f_{\mathrm{o}}\left(\mathbf{v}^{\prime}\right) \chi\left(x, y, v_{x}, v_{y}\right)$

where $0 \leq \chi\left(x, y, v_{x}, v_{y}\right) \leq 1$. If $f_{\mathrm{o}}\left(\mathbf{v}^{\prime}\right)$ is isotropic then energy conservation uniquely determines the original speed $v^{\prime}$,

$v^{\prime}=\sqrt{v^{2}-\frac{2 e V(x, y)}{m_{e}}}$.

Then we can estimate the density inside the potential structure

$$
\begin{aligned}
n(x, y) & =\int d^{2} \mathbf{v} f(x, y, \mathbf{v}) \\
& \leq \int d^{2} \mathbf{v} f_{\mathrm{o}}\left(v^{\prime}\right) \\
& =\int d^{2} \mathbf{v} f_{\mathrm{o}}\left(\sqrt{v^{2}-2 e V(x, y) / m_{e}}\right) \\
& =2 \pi \int_{\sqrt{2 e V / m_{e}}}^{\infty} d v v f_{\mathrm{o}}\left(\sqrt{v^{2}-2 e V(x, y) / m_{e}}\right) \\
& =2 \pi \int_{0}^{\infty} d v^{\prime} v^{\prime} f_{\mathrm{o}}\left(v^{\prime}\right)
\end{aligned}
$$

where we made a change of integration variable back to $v^{\prime}=$ $\sqrt{v^{2}-2 e V / m_{e}}$ so that $v^{\prime} d v^{\prime}=v d v$ and used the fact that $\chi\left(x, y, v_{x}, v_{y}\right) \leq 1$. The last integral in Eq. (26) is, on the other hand, equal to the external plasma electron density $n_{\mathrm{o}}$ because for isotropic distribution $f_{\mathrm{o}}(v)$,

$n_{\mathrm{o}}=\int d^{2} \mathbf{v} f_{\mathrm{o}}(\mathbf{v})=2 \pi \int_{0}^{\infty} d v v f_{\mathrm{o}}(v)$.

Thus we obtain the important and simple result, originally derived by Laframboise and Parker (1973), that if there are no trapped electrons, then the electron density inside a twodimensional potential structure is at most equal to the external plasma density,

$n(x, y) \leq n_{\mathrm{o}}$

provided that the external distribution is isotropic. In fact, non-trapped electrons, once sucked into the potential well, move inside it at speed which is factor $\sqrt{V / V_{\text {th }}}$ higher than their original thermal speed. Since electron thermal energy $e V_{\text {th }}$ is $\sim 12 \mathrm{~V}$ and the potential $V$ is several kilovolts, this factor becomes 10-30. In the outskirts of the potential structure, the electron density is depressed by almost the same factor because of electron number flux conservation. In the inner parts, focussing of electrons towards the attracting tether modifies the pictures. Figure 11 shows results from a small test particle calculation where 4000 electrons were launched into a cylindrically symmetric, $200 \mathrm{~V}$ potential well which has linearly rising walls (constant electric field inside the well). The plot clearly shows how the electron density at the outskirts of the potential well is depressed and the density at the middle does not rise above the background value.

Inequality (28) holds for isotropic external electron distribution. In the solar wind, the electron distribution is not far from being isotropic because the typical thermal speed $\sim 1500 \mathrm{~km} / \mathrm{s}$ is clearly larger than the typical bulk speed $\sim 400 \mathrm{~km} / \mathrm{s}$. Some anisotropy may also result from modest electron temperature anisotropy in the magnetic field aligned system, but generally speaking the electrons are not too far from being isotropic. Thus, the above result (Eq. 28) should be rather well satisfied by solar wind electrons.

\subsection{Thrust from electrosphere model}

Consider again the potential structure ("electrosphere") that forms around the charged tether, under the assumption that all trapped electrons have been removed. The outer boundary of the electrosphere ("electropause") in the sunward direction would be expected to have a potential jump of $\sim 1 \mathrm{kV}$ which stops the solar wind ions. Inside the boundary there are no ions, while the electron density varies from point to point between near zero and $n_{\mathrm{o}}$.

In analogue with magnetopause formation, the electropause on the sunward side is expected to settle at a point where the local electric pressure $(1 / 2) \epsilon_{\mathrm{o}} E^{2}$ balances the solar wind dynamic pressure. If the inside of the electrosphere would be a complete vacuum, the field $E(r)$ would be simply $E_{0}\left(r_{w} / r\right)$ where $E_{0}$ is the field at $r=r_{w}$. To obtain a richer model, however, let us assume that the electrospheric electron density, instead of being zero, is some constant $n_{e}$. Then the radial dependence of the electric field is obtained from Gauß' law,

$E(r)=E_{0}\left(\frac{r_{w}}{r}\right)-\frac{e n_{e} r}{2 \epsilon_{\mathrm{o}}}$.

Imposing the force balance condition

$\frac{1}{2} \epsilon_{\mathrm{o}} E(R)^{2}=P_{\text {dyn }}$

and solving for the electropause subsolar distance $R$ we obtain after some algebra

$R=\frac{2 \sqrt{\epsilon_{\mathrm{o}} P_{\mathrm{dyn}}(1+A / 2-\sqrt{1+A})}}{e n_{e}}$

where $A=e n_{e} E_{0} r_{w} / P_{\text {dyn }}$.

The potential $V(r)$ can be integrated from $E(r)$ :

$$
\begin{aligned}
V(r) & =V_{0}-\int_{r_{w}}^{r} d r^{\prime} E\left(r^{\prime}\right) \\
& =V_{0}-E_{0} r_{w} \ln \left(\frac{r}{r_{w}}\right)-\frac{e n_{e}}{4 \epsilon_{\mathrm{o}}} r^{2}
\end{aligned}
$$


where a very small term proportional to $r_{w}^{2}$ was neglected. Demanding that $V(R)=V_{1}$ where $V_{1} \approx 1 k V$ is the solar wind ion energy we can solve $V_{0}$ as

$V_{0}=V_{1}+E_{0} r_{w} \ln \left(\frac{R}{r_{w}}\right)+\frac{e n_{e}}{4 \epsilon_{\mathrm{o}}} R^{2}$

Equations (31) and (33) determine $R$ and $V_{0}$ as a function of the surface electric field $E_{0}$ and make it easy to produce a parametric plot which shows the dependence of $R$ on $V_{0}$. It is more interesting, however, at this stage to consider the resulting thrust force per unit tether length $d F / d z$. We assume that $d F / d z$ is given by

$\frac{d F}{d z}=K P_{\mathrm{dyn}} R, \quad K=3.09$

(Janhunen and Sandroos, 2007). The thrust is plotted in Fig. 12 for $n_{e}=0$ and $n_{e}=n_{\mathrm{o}}$ which provide an upper and lower thrust limit if trapped electrons are not present. An arbitrarily chosen case $n_{e}=4 n_{\mathrm{o}}$ is also included to demonstrate that the thrust does not decrease too severely even if a significant number of trapped electrons would remain in the system.

It is noteworthy that the new thrust estimates are $\sim 5$ times higher than those published earlier (Janhunen and Sandroos, 2007). The difference between the old and new results is due to the fact that in the new results we assume that trapped electrons are removed by spacecraft-induced orbit scattering and subsequent tether collisions in few minutes after turning on the potential.

Recently, Sanmartín et al. (2008) calculated the tether sheath thickness with high bias voltage in an immobile, unmagnetised plasma. Their Eq. (37) determines implicitly the sheath thickness $r_{s}$ in terms of the tether radius $r_{w}, \lambda_{D e}, T_{e}$ and $V_{0}$ :

$1.53\left[1-2.56\left(\frac{\lambda_{D e}}{r_{s}}\right)^{4 / 5}\right]\left(\frac{r_{s}}{\lambda_{D e}}\right)^{4 / 3} \ln \left(\frac{r_{s}}{r_{w}}\right)=\frac{e V_{0}}{T_{e}}$.

If one solves $r_{s}$ from Eq. (35) numerically, one finds even larger values than our parameter $R$ above. The reason for the difference between $R$ and $r_{s}$ is that the parameter $r_{s}$ of Sanmartín et al. (2008) corresponds to the distance where the potential $V\left(r_{s}\right)$ becomes comparable to $T_{e} \approx 12 \mathrm{eV}$, whereas our $R$ corresponds to the distance where $V(R) \sim V_{1} \approx 1 \mathrm{kV}$. Thus the result of Sanmartín et al. (2008) is consistent with our results.

\section{Discussion, summary and conclusions}

The results of this paper indicate that the thrust of the electric sail could be some 5 times higher than previously reported, of $\sim 500 \mathrm{nN} / \mathrm{m}$ at $1 \mathrm{AU}$ for average solar wind conditions and for reasonable values of the driving voltage. Naturally, from the practical application viewpoint, such increase in the estimated thrust is very significant. An electric sail apparatus

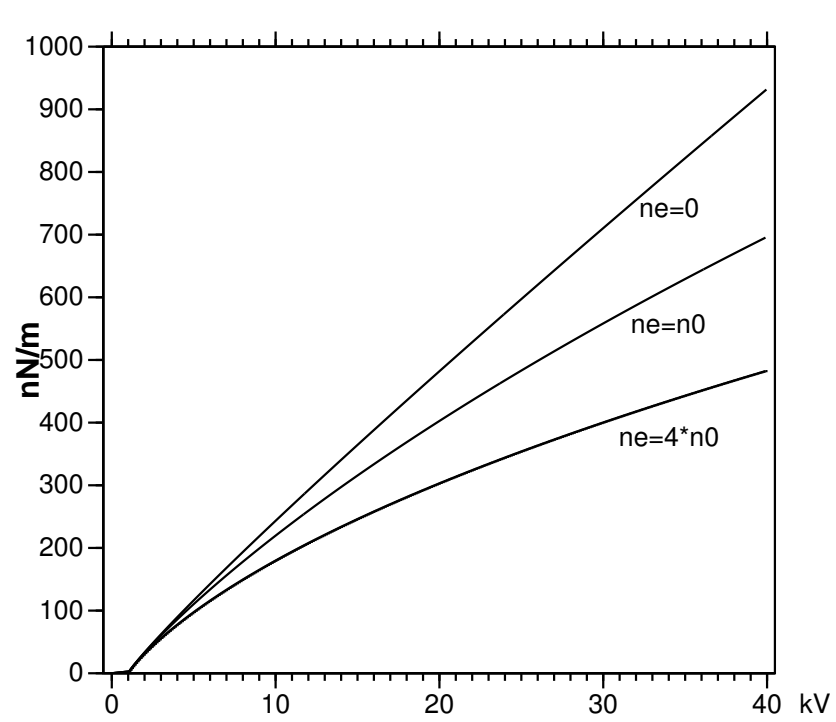

Fig. 12. Thrust per unit tether length (Eq. 34) for different assumed values of the electron density $n_{e}$ inside the electrosphere.

containing e.g. $2000 \mathrm{~km}$ total length of tether (for example, 50 tethers $40 \mathrm{~km}$ long each) could weigh $50-100 \mathrm{~kg}$ (frame, solar panels, high-voltage power source, electron gun, motorised tether reels, various sensors and control processor), of which the tether mass is $10 \mathrm{~kg}$. According to the new results, such a device could produce $\sim 1 \mathrm{~N}$ thrust and produce a specific acceleration of $10-20 \mathrm{~mm} / \mathrm{s}^{2}$. If used to move a $500 \mathrm{~kg}$ payload, for example, the device would produce a $30 \mathrm{~km} / \mathrm{s}$ velocity change over six months. Analysis of applications is, however, left outside the scope of this paper.

The main idea of this paper is that trapped electrons are removed from electric sail tether potential structures because of orbit chaotisation produced by the 3-D potential structure of the spacecraft body. The trapped electron lifetime depends on how wide the potential structure is, but is below $3 \mathrm{~min}$ for structure width below $200 \mathrm{~m}$ (Fig. 10). Thus, one expects that trapped electrons are typically completely or nearly absent. Under the assumption of no trapped electrons, we derived the resulting size of the potential structure from the electropause pressure balance condition (30), keeping the electrospheric electron density $n_{e}$ as a free parameter. We showed that $n_{e} \leq n_{\mathrm{o}}$ should hold. The resulting thrust (Fig. 12) is rather insensitive to the poorly known value of $n_{e}$ when $0<n_{e} \leq n_{\mathrm{o}}$. In reality, $n_{e}$ is not a constant (see Fig. 11), but the result should still fall inbetween the curves marked with $n_{e}=0$ and $n_{e}=n_{\mathrm{o}}$ in Fig. 12. For turning the electrosphere subsolar distance $R$ into a thrust estimate, we used a coefficient $K \approx 3.09$ found in our earlier paper (Janhunen and Sandroos, 2007) from ion test particle calculation using somewhat different functional form for the potential structure.

The theoretical results presented here call for experimental verification. The verification could come from a 
measurement of electrosphere size, thrust force or both in a space or laboratory experiment. Two-dimensional particlein-cell or Vlasov plasma simulations might give a better estimate of the thrust force than the rough analytical calculations presented in this paper. The 2-D simulations would need to be equipped with some kind of trapped electron removal scheme. Because the electron temperature $\sim 12 \mathrm{eV}$ is several thousand times smaller than the depth of the potential well, extra care should be taken into the simulations to avoid spurious trapping by numerical errors.

Although the electric sail plasma physical problem is simple in the sense that only electrostatic forces are involved, the problem spans a wide range in parameter space. The range in energy goes from $12 \mathrm{eV}$ electron temperature to $20 \mathrm{kV}$ tether potential. The spatial scale is from $10 \mu \mathrm{m}$ radius wires to $100 \mathrm{~m}$ wide potential structure and to $20-100 \mathrm{~km}$ long tethers, which gives 7 to 10 orders of magnitude in space. Finally, the timescales start from $0.1 \mathrm{ps}$ needed for an electron to move across a $10 \mu \mathrm{m}$ wire width to several minutes needed to remove the trapped electrons (15-16 orders of magnitude). It is evident from this range of scales that a brute-force simulation approach is not fruitful. Thus, while theory is essential and simulations helpful, experimental studies are crucial in designing the electric sail.

Finally, it is worth remarking that if the electric sail thrust is indeed as large as the estimates presented in this paper indicate, the potential of the electric sail for space transportation in the solar system is enormous. Exploring the potential scientific and commerical applications and implications is, however, outside the scope of this theoretical study.
Acknowledgements. The author thanks Petri Toivanen and Arto Sandroos for many discussions related to the topic and acknowledges the much larger international electric sail team for background support. The work was supported by the Academy of Finland and the Väisälä Foundation.

Topical Editor I. A. Daglis thanks P. Valek and another anonymous referee for their help in evaluating this paper.

\section{References}

Gurevich, A. V.: Distribution of captured particles in a potential well in the absence of collisions, Sov. Phys. JETP, 26(3), 575580, 1968.

Hoyt, R. and Forward, R. L.: Alternate interconnection Hoytether failure resistant multiline tether, US Pat. 6286788 B1, 2001.

Laframboise, J. G. and Parker, L. W.: Probe design for orbit-limited current collection, Phys. Fluids, 16(5), 629-636, 1973.

Janhunen, P.: Electric sail for spacecraft propulsion, J. Propulsion Power, 20(4), 763-764, 2004.

Janhunen, P. and Sandroos, A.: Simulation study of solar wind push on a charged wire: basis of solar wind electric sail propulsion, Ann. Geophys., 25, 755-767, 2007, http://www.ann-geophys.net/25/755/2007/.

Janhunen, P.: Electric sail for producing spacecraft propulsion, US Pat. 11/365875 (pending), 2006.

Janhunen, P.: The electric sail - a new porpulsion method which may enable fast missions to the outer solar system, J. British Interplanetary Soc., 61, 322-325, 2008.

Mengali, G., Quarta, A., and Janhunen, P.: Electric sail performance analysis, J. Spacecraft Rockets, 45, 122-129, 2008.

Mengali, G., Quarta, A. A., and Janhunen, P.: Considerations of electric sailcraft trajectory design, J. British Interplanetery Soc., 61, 326-329, 2008.

Sanmartín, J. R., Choinière, E., Gilchrist, B. E., Ferry, J.-B., and Martínez-Sánchez, M.: Bare-tether sheath and current: comparison of asymptotic theory and kinetic simulations in stationary plasma, IEEE Trans. Plasma Phys., 36, 2851-2858, 2008. 